University of New Orleans

ScholarWorks@UNO

$5-2003$

\title{
Dielectric constant and proton order and disorder in ice Ih: Monte Carlo computer simulations
}

Steven W. Rick

University of New Orleans, srick@uno.edu

Follow this and additional works at: https://scholarworks.uno.edu/chem_facpubs

Part of the Chemistry Commons

\section{Recommended Citation}

Steven W. Rick and A. D. J. Haymet. 2003. " Dielectric constant and proton order and disorder in ice Ih: Monte Carlo computer simulations." Journal of Chemical Physics 118 (20): 9291-9296.

This Article is brought to you for free and open access by the Department of Chemistry at ScholarWorks@UNO. It has been accepted for inclusion in Chemistry Faculty Publications by an authorized administrator of ScholarWorks@UNO.For more information, please contact scholarworks@uno.edu. 


\title{
Dielectric constant and proton order and disorder in ice Ih: Monte Carlo computer simulations
}

\author{
Steven W. Rick ${ }^{\text {a) }}$ \\ Department of Chemistry, University of New Orleans, New Orleans, Louisiana 70148 \\ and Chemistry Department, Southern University of New Orleans, New Orleans, Louisiana 70126
}

A. D. J. Haymet ${ }^{\text {b) }}$

Department of Chemistry, University of Houston, Houston, Texas 77204-5003

(Received 10 December 2002; accepted 26 February 2003)

\begin{abstract}
The dielectric properties of ice Ih are studied using a Monte Carlo algorithm for sampling over proton configurations. The algorithm makes possible the calculation of the dielectric constant and other properties of the proton-disordered crystal. Results are presented for three classical models of water, two commonly used nonpolarizable models (SPC/E and TIP4P) and a polarizable model (TIP4P-FQ). (C) 2003 American Institute of Physics. [DOI: 10.1063/1.1568337]
\end{abstract}

\section{INTRODUCTION}

The high dielectric constant of ice Ih represents one of the many anomalous properties of the condensed phases of water. ${ }^{1,2}$ At the coexistence temperature at 1 atm pressure, ice has a larger dielectric constant than liquid water, even though, as is well known, it has a lower density. The high dielectric constant is indicative of the disorder of the protons present in ice Ih (as well as some other ice phases). The mechanism for proton rearrangement in ice involves both orientational defects (the Bjerrum D and L defects) and ionic defects. ${ }^{3}$ In pure ice, these defects are present in extremely low concentrations, one in $5 \times 10^{6}$ water molecules for Bjerrum defects and $10^{5}$ times less for ionic defects. Computer simulations typically use system sizes of 100-1000 molecules and therefore cannot include these defects at the normally occurring concentrations. In this work, we present an algorithm for sampling efficiently over proton configurations, which yields the calculation of the dielectric constant of ice.

The water molecules are arranged according to the ice rules: water molecules are present as neutral $\mathrm{H}_{2} \mathrm{O}$ and each molecule makes four hydrogen bonds with its four nearest neighbors, two as a hydrogen bond donor and two as an acceptor. If all proton configurations satisfying the ice rules are equally probable, then the resulting entropy difference between the disordered and ordered states is in very good agreement with the observed residual entropy of ice. However, all proton configurations are most likely not energetically equivalent. At temperatures below $100 \mathrm{~K}$, it is believed that a transition occurs to a proton-ordered phase, representing the lowest-energy proton configuration. ${ }^{4}$ The annealing times may be prohibitively long to observe the ordered phase of pure ice, but for ice Ih doped with alkali hydroxides, which decrease the relaxation time, the order-disorder tran-

\footnotetext{
a) Author to whom correspondence should be addressed. Electronic mail: srick@uno.edu

b) Present address: CSIRO Marine Research, GPO Box 1538, Hobart TAS 7001 Australia.
}

sition occurs at $71.6 \mathrm{~K}$, apparently independent of the type and concentration of the dopant. ${ }^{4}$

A general method for generating proton positions in the ice lattice is first to identify a set of water molecules which form a hydrogen-bonded loop and then to shift collectively the protons along the loop. This approach was first used by Rahman and Stillinger, who treated all proton configurations satisfying the ice rules as energetically equivalent. ${ }^{5}$ Subsequent studies of two-dimensional ice by Yanagawa and Nagle $^{6}$ and ice Ih by Barkema and de Boer ${ }^{7}$ used the Monte Carlo method to sample proton configurations according to their energies. These studies placed the oxygen and hydrogen atoms on lattice sites, with no lattice vibrations or distortions. The only degrees of freedom not fixed in these studies were the protons. Here we present an algorithm for generating proton positions, which is general enough to be combined with sampling over the other degrees of freedom. In Sec. II we describe the method and give the details of the simulations. In Sec. III, we present the results for the dielectric constant and proton order parameters of ice for three water models, and in Sec. IV, we summarize our results.

\section{METHODS: THE MOVE ALGORITHM}

To sample over proton configurations with the proper Boltzmann weighting at a specified temperature, we have developed an algorithm involving two steps. The first step is to find a closed hydrogen bonded loop in the ice crystal using a random walk. The second step is to generate new proton positions, satisfying the ice rules, by changing the hydrogen bond pattern. Our algorithm is described as follows:

(1) Find a closed hydrogen-bonded loop.

(a) Randomly select a molecule in the lattice $i$.

(b) Randomly choose one of its four nearest neighbors $j$.

(c) If $j$ is a hydrogen bond donor to $i$, then randomly pick one of the two nearest neighbors of $j$ which are hydrogen bond donors to $j$; otherwise, randomly pick one of the two nearest neighbors of $j$ which are hydrogen bond acceptors to $j$. 

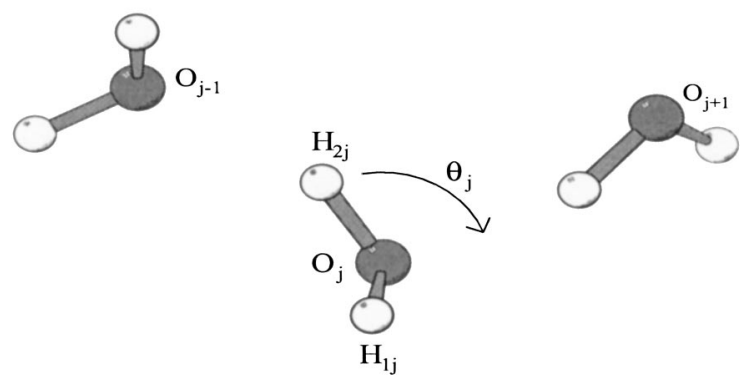

FIG. 1. Illustration of the Monte Carlo algorithm for generating new proton positions by rotation about the $\mathrm{O}_{j}-\mathrm{H}_{1 j}$ axis.

(d) Continue to walk randomly until the path connects back to any spot on the path, not necessarily the first molecule. For finite-size systems, the walk will have to cross itself at some point. For systems with periodic boundary conditions, the closed loop may be a molecule displaced by the side length of the periodic box (a periodic image of the original molecule in the loop).

(2) Generate new proton positions.

(a) For each molecule $j$ on the $N$-molecule loop, rotate the molecule about the $\mathrm{O}_{j}-\mathrm{H}_{1 j}$ axis, where $H_{1 j}$ is the hydrogen atom which is not in the loop. The molecule is rotated by an angle $\theta_{j}$.

(b) The angle $\theta_{j}$ depends on the position of $\mathrm{O}_{j-1}, \mathrm{O}_{j+1}$, $\mathrm{O}_{j}$, and $\mathrm{H}_{1 j}$ (see Fig. 1). It is equal to the angle between the planes formed by $\mathrm{O}_{j-1}, \mathrm{O}_{j}$, and $\mathrm{H}_{1 j}$ and $\mathrm{O}_{j+1}, \mathrm{O}_{j}$, and $\mathrm{H}_{1 j}$. For $j=1$, the $\mathrm{O}_{j-1}$ corresponds to $\mathrm{O}_{N}$, and for $j=\mathrm{N}, \mathrm{O}_{j+1}$ corresponds $\mathrm{O}_{1}$.

The move is accepted based on the standard Monte Carlo or Metropolis algorithm, ${ }^{8}$ with an acceptance probability equal to $\operatorname{Min}\left(1, \mathrm{e}^{\left(E_{a}-E_{b}\right) / k T}\right)$, where $E_{a}$ is the energy before and $E_{b}$ is the energy after the proton move, $k$ is the Boltzmann constant, and $T$ is temperature. The many-proton moves can be combined with smaller amplitude moves of the oxygen and hydrogen atoms using standard Monte Carlo or canonical ensemble (constant $T, V, N$ ) molecular dynamics. Either method is applicable, and we choose to use constanttemperature molecular dynamics because it is more efficient for use with polarizable models. ${ }^{9}$

Step 1, for finding the hydrogen-bonded loop, is the same as methods presented previously for generating proton configurations in ice. ${ }^{5-7}$ The previous studies all used oxygen and hydrogen positions on an otherwise perfect lattice, with no lattice vibrations or molecular rotations. In this case, generating the new proton positions simply involves moving the proton along the line connecting the neighboring oxygen atoms. Due to thermal motion, however, the hydrogens will not necessarily be on the $\mathrm{O}-\mathrm{O}$ line, as illustrated in Fig. 1, so a different method for generating new proton positions is required (step 2 ). The rotation angle $\theta_{j}$ is about $120^{\circ}$, but will vary due to lattice vibrations and nonideal values for the lattice constant ratio $c / a$. Using a rotation angle equal to $120^{\circ}$ gives a vanishingly small acceptance ratio for the proton moves and is not recommended.

Combining the proton Monte Carlo moves with molecular dynamics requires a new set of velocities after each water molecule is rotated. One method is to simply rotate the velocities of each atom along the position. In the standard Verlet algorithm, the positions at the previous time step, rather than the velocities, are used together with the present positions and forces to generate the trajectory. ${ }^{10}$ Using the Verlet algorithm, the previous time step positions for all three atoms are rotated by the same angle $\theta$ around the $\mathrm{O}-\mathrm{H}_{1}$ axis (of the present position). This is equivalent to rotating the velocities and will not change the kinetic energy, or temperature, because only the direction, not the magnitude, is changed. However, the momentum will be changed and each accepted proton move will change the net momentum of the system. To keep the momentum of the system constant, a small modification is required. The velocity of atom $\alpha$ on molecule $i$ is given by

$$
\mathbf{v}_{i \alpha}=\left[\mathbf{r}_{i \alpha}(t)-\mathbf{r}_{i \alpha}(t-\Delta t)\right] / \Delta t,
$$

where $\Delta t$ is the time step, $\mathbf{r}_{i \alpha}(t)$ is the present position, at time $t$, and $\mathbf{r}_{i \alpha}(t-\Delta t)$ is the previous position. If the net momentum is to be conserved, then the contribution to the momentum from the molecules which are being moved must be the same before and after the proton move. For a hydrogen-bonded loop with $N$ molecules, the contribution to momentum from these $N$ molecules is given by

$$
\begin{aligned}
\mathbf{P}_{N}^{a} & =\sum_{i=1}^{N} \sum_{\alpha} m_{\alpha} \mathbf{v}_{i \alpha}^{a} \\
& =\sum_{i=1}^{N} \sum_{\alpha} m_{\alpha}\left[\mathbf{r}_{i \alpha}^{a}(t)-\mathbf{r}_{i \alpha}^{a}(t-\Delta t)\right] / \Delta T,
\end{aligned}
$$

where $\mathbf{v}_{i \alpha}$ is the velocity of atom $\alpha$ of molecule $i, \mathbf{r}_{i \alpha}(t)$ and $\mathbf{r}_{i \alpha}(t-\Delta t)$ are the present and previous positions, respectively, $m_{\alpha}$ is the mass of atom $\alpha$, and $\Delta t$ is the time step. The superscript $a$ indicates variables before rotation. After rotation, the contribution to the momentum from the same $N$ molecules is

$$
\begin{aligned}
\mathbf{P}_{N}^{b} & =\sum_{i=1}^{N} \sum_{\alpha} m_{\alpha} \mathbf{v}_{i \alpha}^{b} \\
& =\sum_{i=1}^{N} \sum_{\alpha} m_{\alpha}\left[\mathbf{r}_{i \alpha}^{b}(t)-\mathbf{r}_{i \alpha}^{b}(t-\Delta t)\right] / \Delta T .
\end{aligned}
$$

In order for $\mathbf{P}_{N}^{b}=\mathbf{P}_{N}^{a}$, the previous positions for all atoms in the loop, after they have been rotated, must be shifted by adding a constant $\Delta \mathbf{R}$. The constant is given by

$$
\Delta \mathbf{R}=\frac{\Delta t}{M} \frac{\left(\mathbf{P}_{N}^{b}-\mathbf{P}_{N}^{a}\right)}{N},
$$

where $M$ is the total mass of the water molecule. This modification will lead to conservation of the momentum, but will change slightly the kinetic energy of the system, at each accepted proton move.

The proton move algorithm is both ergodic and satisfies detailed balance, and therefore can sample over all proton configurations with the proper Boltzmann weighting. The ergodicity of the loop algorithm has been demonstrated previously. ${ }^{7,11}$ In brief, any difference in hydrogen bonded configurations can be represented in terms of different 
hydrogen-bonded loops, and so all configurations can be reached in principle. An earlier study of proton disorder has demonstrated this same point, that all different proton configurations, which obey the ice rules, can be expressed as a number of different loops on the ice lattice. ${ }^{12}$ The detailed balance condition ensures that the probability of generating a configuration is proportional to its Boltzmann weighting. Detailed balance equates the probability of being at a specific state $a$ times the transition probability from $a$ to a new state $b, \Pi_{a b}$, to the probability of being at a specific state $b$ times the transition probability from $b$ to $a$, or

$$
e^{-E_{a} / k T} \Pi_{a b}=e^{-E_{b} / k T} \Pi_{b a} .
$$

For the many-proton move, $\Pi_{a b}$ can be written as the product of three terms, $\Pi_{a b}=\Pi_{a b}^{L} \Pi_{a b}^{R} A_{a b}$, where $\Pi_{a b}^{L}$ is the probability of generating the specific hydrogen-bonded loop (step 1, above), $\Pi_{a b}^{R}$ is the probability for generating the new proton positions in that loop (step 2), and $A_{a b}$ is the acceptance probability for the move. The probability of generating the loop in configuration $a$ from the random walk is the same as generating the loop in configuration $b$, and so $\Pi_{a b}^{L}$ $=\Pi_{b a}^{L}$ (see also Ref. 7) The rotation angle $\theta_{j}$ is defined by the oxygen and hydrogen positions in such a way that the angle that would be used to generate the new proton positions starting from state $a$ is exactly minus the angle that would generate the proton positions from $b$. Therefore $\Pi_{a b}^{R}$ $=\Pi_{b a}^{R}$. The probability of generating a trial move is equal to that of the reverse move. With this condition on $\Pi_{a b}$, detailed balance is satisfied if

$$
e^{-E_{a} / k T} A_{a b}=e^{-E_{b} / k T} A_{b a},
$$

which the standard Metropolis acceptance criteria satisfies.

In the simulations reported here, a many-proton move was attempted every 100 molecular dynamics time steps. The simulations were done using three water different water models: the nonpolarizable SPC/E (Ref. 13) and TIP4P (Ref. 14) models and the polarizable TIP4P-FQ (Ref. 9) model. We use a time step of $1 \mathrm{fs}$ and the SHAKE algorithm for bond constraints. ${ }^{10}$ The models used here are all rigid and classical, but our proton move algorithm is sufficiently general to be able to treat flexible or quantum mechanical ${ }^{15}$ models as well. Long-ranged interactions are treated using Ewald sums, with a screening parameter $\kappa$ equal to $5 / L$, where $L$ is the smallest box side length, and 256 reciprocal space lattice vectors. ${ }^{10}$ The simulations used 128 molecules (except as noted) in the canonical $(T, V, N)$ ensemble by coupling to a Nosé-Hoover thermostat and used a orthorhombic unit cell. The lattice constants were chosen to yield a pressure of 1 atm. The original configuration for all simulations was taken to be the proton-ordered, antiferroelectric structure of Davidson and Morokuma ${ }^{16}$ (except as noted below). This unit cell contains 8 molecules and is replicated 16 times ( 4 in the $x$ direction and 2 in the $y$ and $z$ directions) to give the 128molecule sample. (The $x, y$, and $z$ directions correspond to the $a, b$, and $c$ lattice parameters.) For the TIP4P-FQ model, polarization is treated using fluctuating charges, namely, charges which change in response to different electrostatic environments. The charges are updated in the molecular dynamics simulation using the extended Lagrangian method,

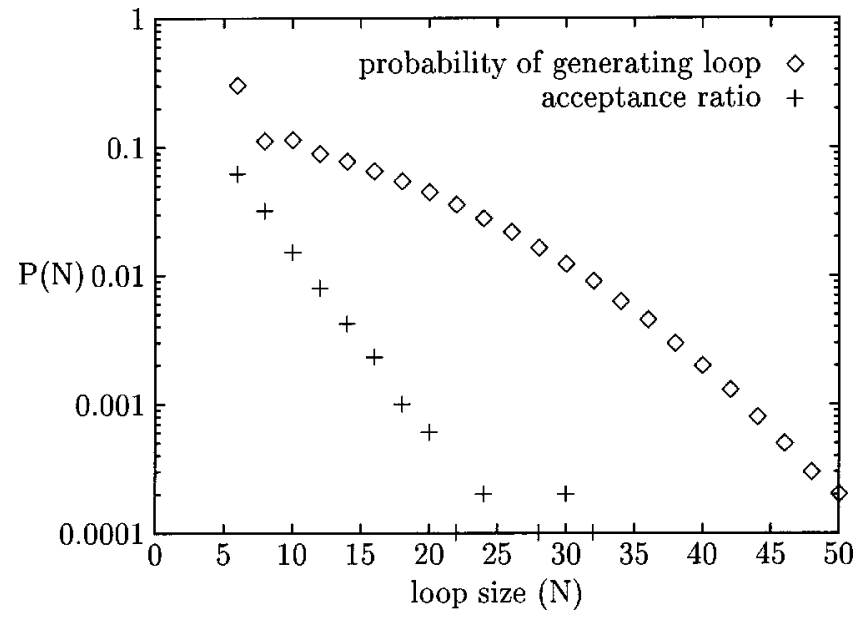

FIG. 2. The probability of generating a loop of size $N$ (diamonds) and the acceptance ratio of a move involving $N$ molecules (crosses) as a function of $N$, shown on a logarithmic scale. The values are for the TIP4P-FQ model at $273 \mathrm{~K}$ with a 128 -molecule $4 \times 2 \times 2$ lattice.

which is an efficient method for keeping the charges close to the ground-state minimum. Each time a many-proton move is attempted, the exact minimum-energy charges are found for the configuration before and after the protons are changed. The simulations are performed over a range of temperature, from 10 to $273 \mathrm{~K}$. Each temperature is simulated for $100 \mathrm{~ns}$, except for some points at lower temperatures, where more simulation data are required to obtain sufficiently small error bars. The TIP4P-FQ model was simulated for $200 \mathrm{~ns}$ at 50 and $100 \mathrm{~K}$ and TIP4P was simulated for 240 ns at $50 \mathrm{~K}$.

The acceptance ratio for the many-proton move depends on temperature and on the water model. For TIP4P, the acceptance ratio ranges from $0.8 \%$ at $50 \mathrm{~K}$ to $5.2 \%$ at $240 \mathrm{~K}$. For TIP4P-FQ, the acceptance ratio ranges from $0.2 \%$ at 100 $\mathrm{K}$ to $2.5 \%$ at $273 \mathrm{~K}$. For SPC/E, the acceptance ratio at 200 $\mathrm{K}$ is $1.3 \%$. The acceptance ratio also depends on the size of the loop. Figure 2 also shows the probability of generating a loop of size $N$. The smallest loop possible on the ice Ih lattice contains 6 molecules and the probability of generating an $\mathrm{N}$-molecule loop through the random walk decreases as $N$ increases. (This probability will depend on the size of the system, depending on how many molecules it takes to reach a periodic image.) The acceptance ratio drops off approximately exponentially with $N$.

\section{RESULTS}

\section{A. Dielectric constant}

The static dielectric constant can be found from

$$
\boldsymbol{\epsilon}=\boldsymbol{\epsilon}_{\infty}+\left(\frac{4 \pi}{3 V k T}\right)\left(\left\langle\boldsymbol{M}^{2}\right\rangle-\langle\boldsymbol{M}\rangle^{2}\right),
$$

where $\boldsymbol{M}$ is the total dipole moment of the system and $\langle\cdots\rangle$ indicates an ensemble average. ${ }^{10}$ For the polarizable model, the optical dielectric constant $\epsilon_{\infty}$ is set equal to $1.592,{ }^{9}$ and for the nonpolarizable TIP4P and SPC/E models, $\epsilon_{\infty}$ equals 
TABLE I. Dielectric constants and $G$ factors as a function of temperature for the TIP4P, TIP4P-FQ, and SPC/E models of water. Numbers in parentheses represent $95 \%$ confidence limits.

\begin{tabular}{lccccl}
\hline \hline Model & $T(\mathrm{~K})$ & $\epsilon$ & $\epsilon_{\perp}$ & $\epsilon_{\|}$ & \multicolumn{1}{c}{$G$} \\
\hline TIP4P-FQ & 273 & $91(3)$ & $90(11)$ & $92(5)$ & $2.63(9)$ \\
TIP4P-FQ & 240 & $100(5)$ & $101(7)$ & $99(13)$ & $2.4(1)$ \\
TIP4P & 240 & $48(1)$ & $47(4)$ & $50(3)$ & $2.54(5)$ \\
TIP4P-FQ & 200 & $130(16)$ & $132(29)$ & $127(13)$ & $2.5(3)$ \\
TIP4P & 200 & $57(3)$ & $58(4)$ & $57(5)$ & $2.5(1)$ \\
SPC/E & 200 & $50(4)$ & $55(1)$ & $40(4)$ & $1.9(2)$ \\
TIP4P-FQ & 150 & $165(26)$ & $173(25)$ & $149(32)$ & $2.2(3)$ \\
TIP4P & 150 & $78(4)$ & $77(7)$ & $80(4)$ & $2.6(1)$ \\
TIP4P-FQ & 100 & $213(51)$ & $192(61)$ & $256(99)$ & $1.7(4)$ \\
TIP4P & 100 & $105(5)$ & $104(12)$ & $108(5)$ & $2.3(1)$ \\
TIP4P-FQ & 50 & $1.71(3)$ & $1.73(3)$ & $1.68(1)$ & 0 \\
TIP4P & 50 & $212(25)$ & $218(45)$ & $201(27)$ & $2.3(3)$ \\
TIP4P-FQ & 10 & $1.70(4)$ & $1.74(6)$ & $1.63(1)$ & 0 \\
TIP4P & 10 & $1.05(1)$ & $1.08(1)$ & $1.00(1)$ & 0 \\
\hline \hline
\end{tabular}

1. The average of $\boldsymbol{M}$ is zero and the $\langle\boldsymbol{M}\rangle^{2}$ term in Eq. (7) can be omitted. The anisotropy of the dielectric constant is given by the components

$$
\boldsymbol{\epsilon}_{\alpha \beta}=\boldsymbol{\epsilon}_{\infty}+\left(\frac{4 \pi}{3 V k T}\right)\left(\left\langle\boldsymbol{M}_{\alpha} \cdot \boldsymbol{M}_{\beta}\right\rangle-\left\langle\boldsymbol{M}_{\alpha}\right\rangle\left\langle\boldsymbol{M}_{\beta}\right\rangle\right) .
$$

The dielectric response is also commonly expressed in terms of the correlation parameter $G$, which equals $\left\langle\boldsymbol{M}^{2}\right\rangle /\left(N_{\text {molec }}\left\langle\boldsymbol{\mu}^{2}\right\rangle\right)$, where $\boldsymbol{\mu}$ is the dipole moment of a water molecule and $N_{\text {molec }}$ is the number of molecules in the system.

The calculated values of the dielectric constants and $G$ are shown in Table I. In agreement with experiment, the calculated dielectric constants decrease with temperature (Fig. 3). At low temperatures, starting with $50 \mathrm{~K}$ for TIP4P-FQ and $10 \mathrm{~K}$ for TIP4P, the protons are frozen in the antiferroelectric ground state and no proton moves are accepted. The low-temperature results suggest that for these potential models the proton-ordered lattice may be the stable

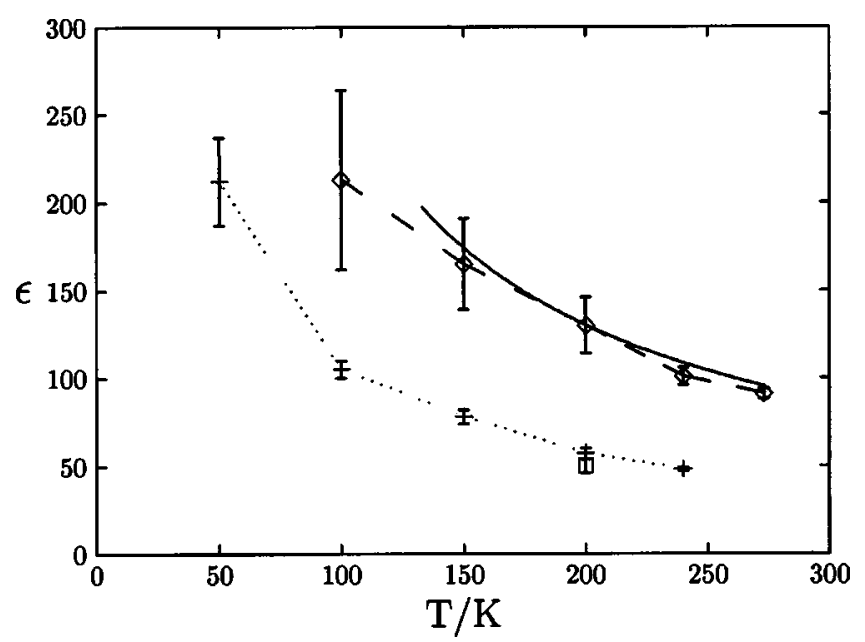

FIG. 3. The calculated dielectric constants of ice Ih for the TIP4P-FQ model (dashed line and diamonds), TIP4P model (dotted line and crosses), and SPC/E model (the square), compared to the experimental values from Ref. 17 (solid line). phase at these temperatures. This would indicate a protonordered phase transition, with a transition temperature between 50 and $100 \mathrm{~K}$ for TIP4P-FQ and 10 and $50 \mathrm{~K}$ for TIP4P. The transition temperature is in the range of the estimates from other theoretical ${ }^{7,17,18}$ and experimental studies, ${ }^{4,19-23}$ although the experimental data on the $\mathrm{KOH}-$ doped ice indicate that the proton-ordered state is ferroelectric. ${ }^{24,25}$ The apparent transition may just be due to sampling problems at low temperatures.

The polarizable TIP4P-FQ model has a higher dielectric constant than the nonpolarizable models and is close to the experimental values. The high-temperature values for $\epsilon$ are similar to the calculated dielectric constants for each of the models in the liquid phase. ${ }^{9,26-30}$ The better agreement with the experimental ice dielectric may be due to the dipole moment of the TIP4P-FQ model, which is about $3.0 \mathrm{D}$ at 273 $\mathrm{K},{ }^{30}$ rather than 2.18 for TIP4P or 2.35 for SPC/E. Onsager and Dupuis ${ }^{31}$ and Rahman and Stillinger ${ }^{5}$ have both shown that a dipole moment of about 3.0 D is consistent with the experimental dielectric constant. This value is also consistent with other theoretical predictions of the dipole moment of a water molecule in ice. ${ }^{32-34}$

The statistical errors in the calculations are too large to resolve differences in the parallel and perpendicular components of $\epsilon$. The measured values show that $\epsilon_{\|}$is greater than $\epsilon_{\perp} \cdot{ }^{3}$ From the calculated values, no clear trend about the relative magnitudes of the two components is apparent and the anisotropy appears to be small. There is a disagreement in the reported experimental anisotropies, ranging from less than $1 \%$ to more than $20 \%$ at $0{ }^{\circ} \mathrm{C} .{ }^{19}$ Theoretical estimates find small anisotropies, less than $1 \%$ (Refs. 7, 35, and 36) (except for Ref. 5). The $G$ factor is less than 3.0 and decreases as the temperature is lowered. For a fully random lattice, $G$ is equal to $3.0 .^{35,36}$ The perfect lattice simulations of Barkema and de Boer also showed that $G$ decreases as the temperature decreases. ${ }^{7}$ The decrease in $G$ indicates that the protons are not distributed randomly.

\section{B. Order parameters}

Hydrogen bonds between two molecules can be characterized by a dihedral angle $\phi$ between the $\mathrm{HOH}$ angle bisector of the hydrogen bond acceptor and the $\mathrm{OH}_{1}$ axis of the hydrogen bond donor $\left(\mathrm{H}_{1}\right.$ is the hydrogen not involved in this hydrogen bond). For hydrogen bonds along the $c$ axis, the dihedral angle can be $60^{\circ}$ (these are termed oblique mirror ${ }^{37}$ ) or $180^{\circ}$ (inverse mirror). For hydrogen bonds not along the $c$ axis, the angle can be $0^{\circ}$ (inverse center) or $120^{\circ}$ (oblique center). The most stable configuration for the water dimer has $\phi$ equal to $180^{\circ}$. This configuration places the hydrogen not involved in the hydrogen bond away from the hydrogens of the other molecule. Based on the dimer energies, the inverse mirror and the oblique center configurations are lower in energy. However, as illustrated by Pitzer and Polissar, next-nearest-neighbor interactions in the ice lattice decrease the energy differences. ${ }^{17}$ We can define order parameters for the ice lattice based on the angle $\phi$ and define $X_{i m}, X_{o m}, X_{i c}$, and $X_{o c}$ as the fraction of hydrogen bonds that are inverse mirror, oblique mirror, inverse center, and oblique center, respectively. Since there are four total hydro- 
TABLE II. Hydrogen bond orientational order parameters, comparing the simulation results to the values for different lattices.

\begin{tabular}{lllllll}
\hline \hline \multicolumn{1}{c}{ Model } & $T(\mathrm{~K})$ & \multicolumn{1}{c}{$X_{i m}$} & \multicolumn{1}{c}{$X_{o m}$} & \multicolumn{1}{c}{$X_{i c}$} & \multicolumn{1}{c}{$X_{o c}$} & $\left(X_{o m}+X_{i c}\right) / 4$ \\
\hline Fully random lattice & & 0.333 & 0.667 & 1.0 & 2.0 & 0.417 \\
Constrained random lattice & & 0.417 & 0.583 & 0.997 & 2.003 & 0.395 \\
Antiferroelectric lattice & & 1.0 & 0.0 & 0.0 & 3.0 & 0 \\
Ferroelectric lattice & & 1.0 & 0.0 & 3.0 & 0.0 & 0.75 \\
TIP4P-FQ & 273 & $0.379(4)$ & $0.621(4)$ & $0.942(1)$ & $2.058(1)$ & $0.391(1)$ \\
TIP4P-FQ & 240 & $0.385(4)$ & $0.615(4)$ & $0.935(7)$ & $2.065(7)$ & $0.388(1)$ \\
TIP4P & 240 & $0.368(3)$ & $0.632(3)$ & $0.950(3)$ & $2.050(3)$ & $0.396(1)$ \\
TIP4P-FQ & 200 & $0.387(4)$ & $0.613(4)$ & $0.92(1)$ & $2.08(1)$ & $0.384(4)$ \\
TIP4P & 200 & $0.374(2)$ & $0.626(3)$ & $0.952(4)$ & $2.048(4)$ & $0.395(1)$ \\
SPC/E & 200 & $0.368(8)$ & $0.60(2)$ & $0.87(2)$ & $2.13(2)$ & $0.40(2)$ \\
TIP4P-FQ & 150 & $0.409(7)$ & $0.591(7)$ & $0.91(1)$ & $2.09(1)$ & $0.374(3)$ \\
TIP4P & 150 & $0.376(4)$ & $0.624(4)$ & $0.948(5)$ & $2.052(5)$ & $0.393(2)$ \\
TIP4P-FQ & 100 & $0.45(2)$ & $0.55(2)$ & $0.87(3)$ & $2.13(3)$ & $0.35(1)$ \\
TIP4P & 100 & $0.389(3)$ & $0.611(3)$ & $0.933(9)$ & $2.067(9)$ & $0.386(2)$ \\
TIP4P-FQ & 50 & 1.0 & 0.0 & 0.0 & 3.0 & 0.0 \\
TIP4P & 50 & $0.42(1)$ & $0.58(1)$ & $0.91(2)$ & $2.09(1)$ & $0.373(3)$ \\
TIP4P-FQ & 10 & 1.0 & 0.0 & 0.0 & 3.0 & 0.0 \\
TIP4P & 10 & 1.0 & 0.0 & 0.0 & 3.0 & 0.0 \\
\hline \hline
\end{tabular}

gen bonds for each molecule and one is along the $c$ axis, $X_{i m}+X_{o m}$ must equal 1 and $X_{i c}+X_{o c}$ must equal 3. The fraction of high-energy hydrogen bonds can be defined as $\left(X_{o m}+X_{i c}\right) / 4$.

The calculated order parameters as a function of temperature are shown on Table II. Also shown are the values for various lattices: the fully random lattice, the random lattice of Hayward and Reimers, generated with the ice rule, zero dipole moment, and zero quadrupole moment constraints, ${ }^{38}$ the antiferroelectric lattice of Davidson and Morokuma, ${ }^{16}$ and the ferroelectric lattice. ${ }^{24,25}$ Note that the HaywardReimers lattice has values that are slightly different from the fully random lattice for the order parameters $X_{i m}$ and $X_{o m}$. The constraints apparently cause $X_{o m}$ to not equal $2 X_{i m}$. (The values are for the $6 \times 4 \times 4$ lattice with 768 molecules. ${ }^{38}$ ) The values from the simulations show that the proton positions are not fully random and that the fraction of high-energy hydrogen bonds, $\left(X_{o m}+X_{i c}\right) / 4$, decreases with temperature. The order parameters approach the antiferroelectric and not the ferroelectric values. This trend is consistent with the results of Barkema and de Boer. ${ }^{7}$

We can write

$$
\frac{X_{i m}}{X_{o m}}=\frac{1}{2} e^{-\left(\left\langle E_{i m}\right\rangle-\left\langle E_{o m}\right\rangle\right) / k T}
$$

and

$$
\frac{X_{i c}}{X_{o c}}=\frac{1}{2} e^{-\left(\left\langle E_{i c}\right\rangle-\left\langle E_{o c}\right\rangle\right) / k T},
$$

where $\left\langle E_{\alpha}\right\rangle$ is the average energy of hydrogen bonds of type $\alpha$, at a particular temperature and density, and the factor of $1 / 2$ is from the fact that there are twice as many oblique as inverse hydrogen bonds. If the temperature dependence on the average energy is small, then a plot of $\ln \left(X_{i m} / X_{o m}\right)$ and $\ln \left(X_{i c} / X_{o c}\right)$ versus $1 / T$ should give a straight line, with the slope indicating the energy difference between the two types of hydrogen bonds (see Fig. 4). The slopes give a very small energy difference between the two different types of hydro- gen bonds. For TIP4P-FQ, $\left\langle E_{\text {im }}\right\rangle-\left\langle E_{\text {om }}\right\rangle$ equals $0.08 \mathrm{kcal} /$ $\mathrm{mol}$, and for TIP4P, it equals $0.03 \mathrm{kcal} / \mathrm{mol}$. The values for $\left\langle E_{i c}\right\rangle-\left\langle E_{o c}\right\rangle$ are $-0.04 \mathrm{kcal} / \mathrm{mol}$ for TIP4P-FQ and $-0.008 \mathrm{kcal} / \mathrm{mol}$ for TIP4P. The differences are smaller for the TIP4P model. Both models are an order of magnitude smaller than the estimates of Pitzer and Polissar, which only take into account some of the nearest neighbor interactions. ${ }^{17}$

\section{Dependence on system size and initial conditions}

Previous calculations of the dielectric constant of liquid water have been done for a variety of system sizes, including
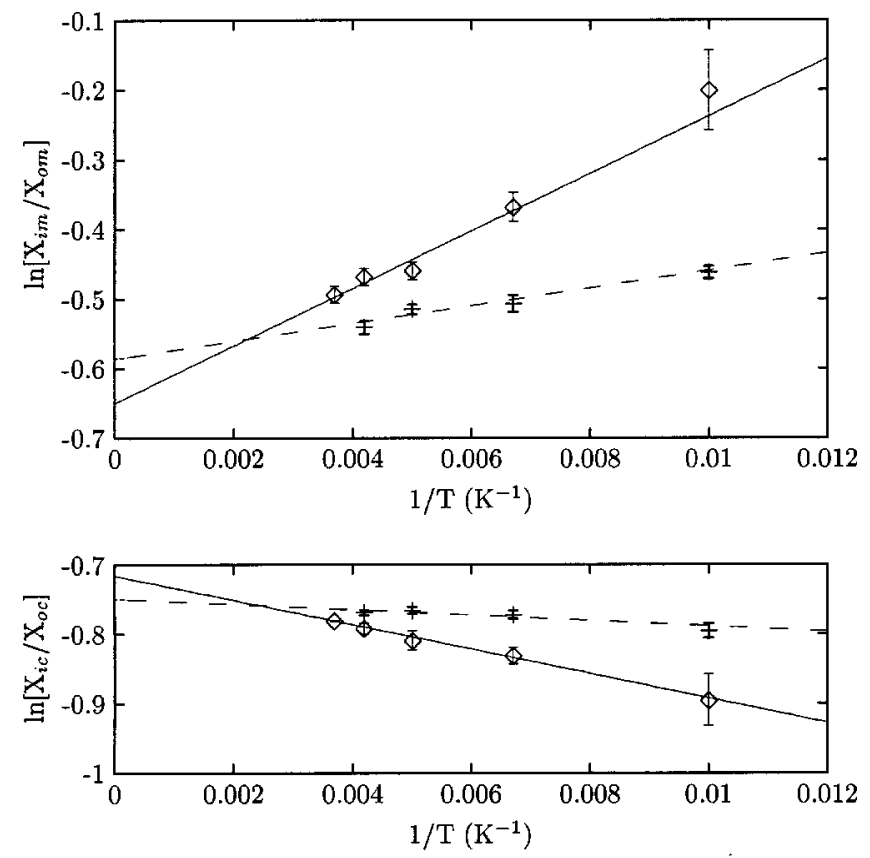

FIG. 4. The energy difference between the two types of hydrogen bonds: the $\ln \left[X_{i m} / X_{o m}\right]$ (top) and $\ln \left[X_{i c} / X_{o c}\right]$ (bottom) as a function of inverse temperature for the TIP4P-FQ (solid line and diamonds) and TIP4P (dashed lines and crosses) models of water. 
systems smaller than 128 molecules, ${ }^{39}$ and there is no observable strong dependence on the system size. For the solid, sampling over fluctuations of $\boldsymbol{M}$ requires accepting many proton moves that are large enough to reach across the simulation box. The $4 \times 2 \times 2$ box with 128 molecules is 8 molecules wide in each direction. Adding a unit cell in each direction increases the width by two molecules in the $x$ direction and by four in the $y$ and $z$ directions. To sample fluctuations in larger size boxes, proton moves with more molecules are required. The bigger molecule loops are accepted with lower probabilities (see Fig. 2) and more attempted moves will be necessary. We have performed preliminary simulations of a larger system (a $5 \times 3 \times 3$ box with 360 molecules) starting with a proton-ordered antiferroelectric lattice and a disordered lattice of Hayward and Reimers. ${ }^{38}$ Both systems were simulated for $50 \mathrm{~ns}$ and the results for the dielectric constant $(88 \pm 41$ with the protonordered initial lattice and $110 \pm 58$ with the disordered initial lattice) are, within the large error bars, the same as the 128molecule result $(91 \pm 3$; see Table I). The two can be averaged together to give $99 \pm 36$ for the 360 -molecule box with the same total simulation time as the 128-molecule box, but with much larger error bars.

\section{CONCLUSIONS}

A Monte Carlo algorithm for sampling over proton configurations yields a method for calculating the dielectric constant of ice. The results for three water models (SPC/E, TIP4P, and TIP4P-FQ) give values for the dielectric constant which are consistent with the values that these models give for the liquid phase. The method can calculate the dielectric constant at temperatures where it has not yet been measured for pure ice. At low temperatures, around $50 \mathrm{~K}$, there is evidence of a transition to a proton-ordered phase. An examination of proton order parameters indicates that the disordered phase is not completely random with a preference for the energetically lower water orientations.

\section{ACKNOWLEDGMENTS}

One of the authors (S.W.R.) gratefully acknowledges support from the Louisiana Board of Regents Support Fund under Contract No. LEQSF(2001-04)-RD-A-42 and the National Science Foundation under Contract No. CHE0213488. A.D.J.H. gratefully acknowledges support from the Welch Foundation.
${ }^{1}$ N. H. Fletcher, The Physics of Rainclouds (Cambridge University Press, Cambridge, England, 1962).

${ }^{2} \mathrm{~N}$. Fletcher, The Chemical Physics of Ice (Cambridge University Press, Cambridge, England, 1970).

${ }^{3}$ D. Eisenberg and W. Kauzmann, The Structure and Properties of Water (Oxford University Press, New York, 1969).

${ }^{4}$ M. Tyagi and S. S. N. Murthy, J. Phys. Chem. A 106, 5072 (2002).

${ }^{5}$ A. Rahman and F. H. Stillinger, J. Chem. Phys. 57, 4009 (1972).

${ }^{6}$ A. Yanagawa and J. F. Nagle, Chem. Phys. 43, 329 (1979).

${ }^{7}$ G. T. Barkema and J. de Boer, J. Chem. Phys. 99, 2059 (1993).

${ }^{8}$ N. Metropolis, A. Rosenbluth, M. Rosenbluth, A. Teller, and E. Teller, J. Chem. Phys. 21, 1087 (1953).

${ }^{9}$ S. W. Rick, S. J. Stuart, and B. J. Berne, J. Chem. Phys. 101, 6141 (1994).

${ }^{10}$ M. P. Allen and D. J. Tildesley, Computer Simulation of Liquids (Oxford University Press, Oxford, 1987).

${ }^{11}$ G. T. Barkema and M. E. J. Newman, Phys. Rev. E 57, 1155 (1998).

${ }^{12}$ J. F. Nagle, J. Math. Phys. 7, 1484 (1964).

${ }^{13}$ H. J. C. Berendsen, J. R. Grigera, and T. P. Straatsma, J. Phys. Chem. 91, 6269 (1987).

${ }^{14}$ W. L. Jorgensen, J. Chandrasekhar, J. D. Madura, R. W. Impey, and M. L. Klein, J. Chem. Phys. 79, 926 (1983).

${ }^{15}$ Quantization of the hydrogen atoms could be treated using path integral methods.

${ }^{16}$ E. R. Davidson and K. Morokuma, J. Chem. Phys. 81, 3741 (1984).

${ }^{17}$ K. S. Pitzer and J. Polissar, J. Phys. Chem. 60, 1140 (1956).

${ }^{18}$ I. Minagawa, J. Phys. Soc. Jpn. 50, 3669 (1981).

${ }^{19}$ G. P. Johari and E. Whalley, J. Chem. Phys. 75, 1333 (1981).

${ }^{20}$ O. Wörz and R. H. Cole, J. Chem. Phys. 51, 1546 (1969).

${ }^{21}$ S. Kawada and J. Niinuma, J. Phys. Soc. Jpn. 43, 715 (1977).

${ }^{22}$ Y. Tajima, T. Matsuo, and H. Suga, J. Phys. Chem. Solids 35, 1135 (1984).

${ }^{23}$ O. Yamamuro, N. Kuratomi, T. Matsuo, and H. Suga, Solid State Commun. 73, 317 (1990).

${ }^{24}$ A. J. Leadbetter, R. C. Ward, J. W. Clark, P. A. Tucker, T. Matsuo, and H. Suga, J. Chem. Phys. 82, 424 (1985).

${ }^{25}$ S. M. Jackson, V. M. Nield, R. M. Whitworth, M. Orguro, and C. C. Wilson, J. Phys. Chem. B 101, 6142 (1997).

${ }^{26}$ M. Neumann, J. Chem. Phys. 85, 1567 (1986).

${ }^{27}$ M. Rami Ready and M. Berkowitz, Chem. Phys. Lett. 155, 173 (1989).

${ }^{28}$ H. E. Alper and R. M. Levy, J. Chem. Phys. 91, 1242 (1989).

${ }^{29}$ Y. Guissani and B. Guillot, J. Chem. Phys. 98, 8221 (1993).

${ }^{30}$ S. W. Rick, J. Phys. Chem. 114, 2276 (2001).

${ }^{31}$ L. Onsager and M. Dupuis, in Electrolytes, edited by B. Pesce (Pergamon, Oxford, 1962), p. 27.

${ }^{32}$ E. R. Batista, S. S. Xantheas, and H. Jónsson, J. Chem. Phys. 109, 4546 (1998).

${ }^{33}$ E. R. Batista, S. S. Xantheas, and H. Jónsson, J. Chem. Phys. 112, 3285 (2000).

${ }^{34}$ L. Delle Site, A. Alavi, and R. M. Lynden-Bell, Mol. Phys. 96, 1683 (1999).

${ }^{35}$ J. F. Nagle, J. Chem. Phys. 61, 883 (1974).

${ }^{36}$ D. J. Adams, J. Phys. C 17, 4063 (1984).

${ }^{37}$ N. Bjerrum, Science 115, 385 (1952).

${ }^{38}$ J. A. Hayward and J. R. Reimers, J. Chem. Phys. 106, 1518 (1997).

${ }^{39}$ J. Anderson, J. J. Ullo, and S. Yip, J. Chem. Phys. 87, 1726 (1987). 